\title{
Existence of Positive Periodic Solutions for Periodic Neutral Lotka-Volterra System with Distributed Delays and Impulses
}

\author{
Zhenguo Luo ${ }^{1,2}$ and Liping $\mathrm{Luo}^{2}$ \\ ${ }^{1}$ Department of Mathematics, National University of Defense Technology, Changsha 410073, China \\ ${ }^{2}$ Department of Mathematics, Hengyang Normal University, Hengyang, Hunan 421008, China
}

Correspondence should be addressed to Zhenguo Luo; robert186@163.com

Received 20 April 2013; Accepted 13 May 2013

Academic Editor: Norio Yoshida

Copyright (c) 2013 Z. Luo and L. Luo. This is an open access article distributed under the Creative Commons Attribution License, which permits unrestricted use, distribution, and reproduction in any medium, provided the original work is properly cited.

By using a fixed-point theorem of strict-set-contraction, we investigate the existence of positive periodic solutions for a class of the following impulsive neutral Lotka-Volterra system with distributed delays: $x_{i}^{\prime}(t)=x_{i}(t)\left[r_{i}(t)-\sum_{j=1}^{n} a_{i j}(t) x_{j}(t)-\right.$ $\left.\sum_{j=1}^{n} b_{i j}(t) \int_{-\tau_{i j}}^{0} f_{i j}(\xi) x_{j}(t+\xi) d \xi-\sum_{j=1}^{n} c_{i j}(t) \int_{-\sigma_{i j}}^{0} g_{i j}(\xi) x_{j}^{\prime}(t+\xi) d \xi\right], \Delta x_{i}\left(t_{k}\right)=-I_{i k}\left(x_{i}\left(t_{k}\right)\right), i=1,2, \ldots, n, k=1,2, \ldots$ Some verifiable criteria are established easily.

\section{Introduction}

It is well known that natural environments are physically highly variable, and in response, birth rates, death rates, and other vital rates of populations vary greatly in time. Theoretical evidence suggests that many population and community patterns represent intricate interactions between biology and variation in the physical environment (see [14]). Thus, the focus in theoretical models of population and community dynamics must be not only on how populations depend on their own population densities or the population densities of other organisms, but also on how populations change in response to the physical environment. It is reasonable to study the models of population with periodic coefficients. In addition to the theoretical and practical significance, the Lotka-Volterra model is one of the famous models for dynamics of population; therefore it has been studied extensively [5-9]. In view of the above effects, by applying a fixed-point theorem of strict-set-contraction, $\mathrm{Li}$ [10] established criteria to guarantee the existence of positive periodic solutions of the following neutral Lotka-Volterra system with distributed delays:

$$
x_{i}^{\prime}(t)=x_{i}(t)\left[a_{i}(t)-\sum_{j=1}^{n} b_{i j}(t) \int_{-T_{i j}}^{0} K_{i j}(\theta) x_{j}(t+\theta) d \theta\right.
$$

$$
\begin{array}{r}
\left.-\sum_{j=1}^{n} c_{i j}(t) \int_{-\widehat{T}_{i j}}^{0} \widehat{K}_{i j}(\theta) x_{j}^{\prime}(t+\theta) d \theta\right], \\
i=1,2, \ldots, n,
\end{array}
$$

where $a_{i}, b_{i j}, c_{i j} \in C\left(R, R^{+}\right)(i, j=1,2, \ldots, n)$ are $\omega$ periodic functions and $T_{i j}, \widehat{T}_{i j} \in(0,+\infty)(i, j=1,2, \ldots, n)$ and $K_{i j}, \widehat{K}_{i j} \in\left(R, R^{+}\right)$satisfying $\int_{-T_{i j}}^{0} K_{i j}(\theta) d \theta=1$, $\int_{-\widehat{T}_{i j}}^{0} \widehat{K}_{i j}(\theta) d \theta, i, j=1,2, \ldots, n$. On the other side, birth of many species is an annual birth pulse or harvesting. To have a more accurate description of many mathematical ecology systems, we need to consider the use of impulsive differential equations [11-13]. Some qualitative properties such as oscillation, periodicity, asymptotic behavior, and stability properties have been investigated extensively by many authors over the past few years [14-18]. However, to our knowledge, there are few published papers discussing the existence of periodic solutions for neutral Lotka-Volterra system with distributed delays and impulses. In this paper, we are concerned with the following neutral Lotka-Volterra system with distributed delays and impulses: 


$$
\begin{array}{r}
x_{i}^{\prime}(t)=x_{i}(t)\left[r_{i}(t)-\sum_{j=1}^{n} a_{i j}(t) x_{j}(t)\right. \\
-\sum_{j=1}^{n} b_{i j}(t) \int_{-\tau_{i j}}^{0} f_{i j}(\xi) x_{j}(t+\xi) d \xi \\
\left.-\sum_{j=1}^{n} c_{i j}(t) \int_{-\sigma_{i j}}^{0} g_{i j}(\xi) x_{j}^{\prime}(t+\xi) d \xi\right], \\
i=1,2, \ldots, n, t \neq t_{k}, \\
\Delta x_{i}\left(t_{k}\right)=-I_{i k}\left(x_{i}\left(t_{k}\right)\right), \quad i=1,2, \ldots, n, k=1,2, \ldots,
\end{array}
$$

where $r_{i}, a_{i j}, b_{i j}, c_{i j} \in C\left(R, R^{+}\right)(i, j=1,2, \ldots, n)$ are $\omega$ periodic functions and $\tau_{i j}, \sigma_{i j} \in(0,+\infty)(i, j=1,2, \ldots, n)$ and $f_{i j}, g_{i j} \in C\left(R, R^{+}\right)$satisfying $\int_{-\tau_{i j}}^{0} f_{i j}(\xi) d \xi=1$, $\int_{-\sigma_{i j}}^{0} g_{i j}(\xi) d \xi=1, i, j=1,2, \ldots, n$. Moreover, $\Delta x\left(t_{k}\right)=$ $x\left(t_{k}^{+}\right)-x\left(t_{k}\right)$ (here $x\left(t_{k}^{+}\right)$represents the right limit of $x(t)$ at the point $\left.t_{k}\right), I_{i k} \in C\left(R^{+}, R^{+}\right)$; that is, $x$ changes decreasingly suddenly at times $t_{k} ; \omega>0$ is a constant, $R=$ $(-\infty,+\infty), R^{+}=[0,+\infty)$. We assume that there exists an integer $q>0$ such that $t_{k+q}=t_{k}+\omega, I_{i(k+q)}=I_{i k}$, where $0<t_{1}<t_{2}<\cdots<t_{q}<\omega$.

The main purpose of this paper is by using a fixedpoint theorem of strict-set-contraction $[19,20]$ to establish new criteria to guarantee the existence of positive periodic solutions of the system (2).

For convenience, we introduce the notation

$$
\begin{gathered}
f^{M}=\max _{t \in[0, \omega]}\{|f(t)|\}, \quad f^{L}=\min _{t \in[0, \omega]}\{|f(t)|\}, \\
\gamma_{i}=\lim _{u \rightarrow 0} \sup _{t \leq t_{k} \leq t+\omega} \frac{I_{i k}(u)}{u}, \quad i=1,2, \ldots, n, \\
\delta_{i}=e^{-\int_{0}^{\omega} r_{i}(t) d t}, \quad i=1,2, \ldots, n, \\
\alpha_{i j}=\int_{0}^{\omega}\left[\delta_{j} a_{i j}(t)-b_{i j}(t)-c_{i j}(t)\right] d t, \\
\beta_{i j}=\int_{0}^{\omega}\left[a_{i j}(t)+b_{i j}(t)+c_{i j}(t)\right] d t, \quad i, j=1,2, \ldots, n,
\end{gathered}
$$

where $f$ is a continuous $\omega$-periodic function.

Throughout this paper, we assume that

$$
\begin{aligned}
& \left(A_{1}\right) \delta_{i}=e^{-\int_{0}^{\omega} r_{i}(t) d t}<1, i=1,2, \ldots, n ; \\
& \left(A_{2}\right) \delta_{j} a_{i j}(t)-b_{i j}(t)-c_{i j}(t) \geq 0, i, j=1,2, \ldots, n ; \\
& \left(A_{3}\right) \begin{array}{l}
\left(1+r_{i}^{L}\right)\left(\delta_{i}^{2} /\left(1-\delta_{i}\right)\right) \alpha_{i j} \geq \max _{t \in[0, \omega]}\left\{a_{i j}(t)+b_{i j}(t)+\right. \\
\left.c_{i j}(t)\right\}, i, j=1,2, \ldots, n ;
\end{array} \\
& \left(A_{4}\right)\left(r_{i}^{M}-1\right)\left(1 / \delta_{i}\left(1-\delta_{i}\right)\right) \beta_{i j} \leq \min _{t \in[0, \omega]}\left\{\delta_{j} a_{i j}(t)-b_{i j}(t)-\right. \\
& \left.\quad c_{i j}(t)\right\}, i, j=1,2, \ldots, n ; \\
& \left(A_{5}\right) \max _{1 \leq i \leq n}\left\{\sum_{j=1}^{n} c_{i j}^{M}\right\} \leq \min _{1 \leq i \leq n}\left\{\left(\delta_{i}^{2} /\left(1-\delta_{i}\right)\right)\right. \\
& \left.\min _{1 \leq j \leq n}\left\{\alpha_{i j}\right\}\right\} .
\end{aligned}
$$

The paper is organized as follows. In Section 2, we give some definitions and lemmas to prove the main results of this paper. In Section 3, by using a fixed-point theorem of strictset-contraction, we established some criteria to guarantee the existence of at least one positive periodic solution of system (2). Finally, in Section 4, we give an example to show the validity of our result.

\section{Preliminaries}

In order to obtain the existence of a periodic solution of system (2), we first introduce some definitions and lemmas.

Definition 1 (see [13]). A function $x_{i}: R \rightarrow(0,+\infty)$ is said to be a positive solution of (2), if the following conditions are satisfied:

(a) $x_{i}(t)$ is absolutely continuous on each $\left(t_{k}, t_{k+1}\right)$;

(b) for each $k \in Z_{+}, x_{i}\left(t_{k}^{+}\right)$and $x_{i}\left(t_{k}^{-}\right)$exist and $x_{i}\left(t_{k}^{-}\right)=$ $x_{i}\left(t_{k}\right)$

(c) $x_{i}(t)$ satisfies the first equation of (2) for almost everywhere in $R$ and $x_{i}\left(t_{k}\right)$ satisfies the second equation of (2) at impulsive point $t_{k}, k \in Z_{+}$.

Definition 2 (see [21]). Let $X$ be a real Banach space and $E$ a closed, nonempty subset of $X . E$ is a cone provided

(i) $\alpha x+\beta y \in E$ for all $x, y \in E$ and all $\alpha, \beta \geq 0$,

(ii) $x,-x \in E$ imply $x=0$.

Definition 3 (see [21]). Let $A$ be a bounded subset in $X$. Define

$$
\begin{gathered}
\alpha_{X}(A)=\inf \{\delta>0 \text { : there is a finite number } \\
\text { of subsets } A_{i} \subset A \text { such that } \\
\left.A=\bigcup_{i} A_{i} \text { and } \operatorname{diam}\left(A_{i}\right) \leq \delta\right\},
\end{gathered}
$$

where $\operatorname{diam}\left(A_{i}\right)$ denotes the diameter of the set $A_{i}$, obviously, $0 \leq \alpha_{X}(A)<\infty$. So $\alpha_{X}(A)$ is called the Kuratowski measure of noncompactness of $X$.

Definition 4 (see [21]). Let $X, Y$ be two Banach spaces and $D \subset X$, a continuous and bounded map $T: D \rightarrow Y$, is called $k$-set contractive if, for any bounded set $S \subset D$, we have

$$
\alpha_{Y}(T(S)) \leq k \alpha_{X}(S)
$$

$\phi$ is called strict-set-contractive if it is $k$-set-contractive for some $0 \leq k<1$.

Definition 5 (see [22]). The set $F \in P C_{\omega}$ is said to be quasiequicontinuous in $[0, \omega]$ if for any $\epsilon>0$ there exists $\delta>0$ such that if $x \in F, k \in N^{+}, t_{1}, t_{2} \in\left(t_{k-1}, t_{k}\right) \cap[0, \omega]$, and $\mid t_{1}-$ $t_{2} \mid<\delta$, then $\left|x\left(t_{1}\right)-x\left(t_{2}\right)\right|<\epsilon$. 
Lemma 6 (see [22]). The set $F \subset P C_{\omega}$ is relatively compact if and only if

(1) $F$ is bounded; that is, $\|x\| \leq M$, for each $x \in F$, and some $M>0$;

(2) $F$ is quasi-equicontinuous in $[0, \omega]$.

Lemma 7. $x_{i}(t)$ is an w-periodic solution of (2) is equivalent to $x_{i}(t)$ is an $\omega$-periodic solution of the following equation:

$$
\begin{aligned}
x_{i}(t)= & \int_{t}^{t+\omega} G_{i}(t, s) x_{i}(s) \\
& \times\left[\sum_{j=1}^{n} a_{i j}(s) x_{j}(s)+\sum_{j=1}^{n} b_{i j}(s) \int_{-\tau_{i j}}^{0} f_{i j}(\xi) x_{j}(s+\xi) d \xi\right. \\
& \left.\quad+\sum_{j=1}^{n} c_{i j}(s) \int_{-\sigma_{i j}}^{0} g_{i j}(\xi) x_{j}^{\prime}(s+\xi) d \xi\right] d s \\
& +\sum_{t \leq t_{k}<t+\omega} G_{i}\left(t, t_{k}\right) I_{i k}\left(x_{i}\left(t_{k}\right)\right),
\end{aligned}
$$

where

$$
G_{i}(t, s)=\frac{e^{-\int_{t}^{s} r_{i}(\theta) d \theta}}{1-e^{-\int_{0}^{\omega} r_{i}(\theta) d \theta}}, \quad s \in[t, t+\omega], i=1,2, \ldots, n .
$$

Proof. Assume that $x_{i}(t) \in X, i=1,2, \ldots, n$, is a periodic solution of (2). Then, we have

$$
\begin{gathered}
\frac{d}{d t}\left[x_{i}(t) e^{-\int_{0}^{t} r_{i}(\theta) d \theta}\right] \\
=e^{-\int_{0}^{t} r_{i}(\theta) d \theta} x_{i}(t) \\
\times\left[-\sum_{j=1}^{n} a_{i j}(t) x_{j}(t)-\sum_{j=1}^{n} b_{i j}(t) \int_{-\tau_{i j}}^{0} f_{i j}(\xi) x_{j}(t+\xi) d \xi\right. \\
\left.-\sum_{j=1}^{n} c_{i j}(t) \int_{-\sigma_{i j}}^{0} g_{i j}(\xi) x_{j}^{\prime}(t+\xi) d \xi\right], \\
t \neq t_{k}, i=1,2, \ldots, n .
\end{gathered}
$$

Integrating the previous equation with $[t, t+\omega]$, we can have

$$
\begin{gathered}
\left.x_{i}(s) e^{-\int_{0}^{s} r_{i}(\theta) d \theta}\right|_{t} ^{t_{m_{1}}+n \omega}+\left.x_{i}(s) e^{-\int_{0}^{s} r_{i}(\theta) d \theta}\right|_{t_{m_{1}}+n \omega} ^{t_{m_{2}}+n \omega} \\
+\cdots+\left.x_{i}(s) e^{-\int_{0}^{s} r_{i}(\theta) d \theta}\right|_{t_{m_{q}}+n \omega} ^{t+\omega} \\
=\int_{t}^{t+\omega} e^{-\int_{0}^{s} r_{i}(\theta) d \theta} x_{i}(s) \\
\times\left[-\sum_{j=1}^{n} a_{i j}(s) x_{j}(s)-\sum_{j=1}^{n} b_{i j}(s) \int_{-\tau_{i j}}^{0} f_{i j}(\xi) x_{j}(s+\xi) d \xi\right. \\
\left.\quad-\sum_{j=1}^{n} c_{i j}(s) \int_{-\sigma_{i j}}^{0} g_{i j}(\xi) x_{j}^{\prime}(s+\xi) d \xi\right] d s,
\end{gathered}
$$

where $t_{m_{k}}+n \omega \in(t, t+\omega), m_{k} \in\{1,2, \ldots, q\}, k=$ $1,2, \ldots, q, n \in Z_{+}$. Therefore,

$$
\begin{aligned}
& x_{i}(t) e^{-\int_{0}^{t} r_{i}(\theta) d \theta}\left[1-e^{-\int_{t}^{t+\omega} r_{i}(\theta) d \theta}\right] \\
& +\sum_{t \leq t_{k}<t+\omega} \Delta x_{i}\left(t_{m_{k}}\right) e^{-\int_{0}^{t_{m_{k}}+n \omega} r_{i}(\theta) d \theta} \\
& =\int_{t}^{t+\omega} e^{-\int_{0}^{s} r_{i}(\theta) d \theta} x_{i}(s) \\
& \quad \times\left[\sum_{j=1}^{n} a_{i j}(t) x_{j}(t)+\sum_{j=1}^{n} b_{i j}(s) \int_{-\tau_{i j}}^{0} f_{i j}(\xi) x_{j}(s+\xi) d \xi\right. \\
& \left.\quad+\sum_{j=1}^{n} c_{i j}(s) \int_{-\sigma_{i j}}^{0} g_{i j}(\xi) x_{j}^{\prime}(s+\xi) d \xi\right] d s,
\end{aligned}
$$

which can be transformed into

$$
\begin{aligned}
x_{i}(t)= & \int_{t}^{t+\omega} G_{i}(t, s) x_{i}(s) \\
& \times\left[\sum_{j=1}^{n} a_{i j}(s) x_{j}(s)+\sum_{j=1}^{n} b_{i j}(s) \int_{-\tau_{i j}}^{0} f_{i j}(\xi) x_{j}(s+\xi) d \xi\right. \\
& \left.\quad+\sum_{j=1}^{n} c_{i j}(s) \int_{-\sigma_{i j}}^{0} g_{i j}(\xi) x_{j}^{\prime}(s+\xi) d \xi\right] d s \\
& +\sum_{t \leq t_{k}<t+\omega} G_{i}\left(t, t_{k}\right) I_{i k}\left(x_{i}\left(t_{k}\right)\right), \quad i=1,2, \ldots, n .
\end{aligned}
$$


Thus, $x_{i}$ is a periodic solution of (6). If $x_{i}(t) \in E, i=$ $1,2, \ldots, n$, is a periodic solution of (6), for any $t=t_{k}$, from (6) we have

$$
\begin{aligned}
& x^{\prime}(t)=\frac{d}{d t}\left\{\int_{t}^{t+\omega} G_{i}(t, s) x_{i}(s)\right. \\
& \times\left[\sum_{j=1}^{n} a_{i j}(s) x_{j}(s)\right. \\
& +\sum_{j=1}^{n} b_{i j}(s) \int_{-\tau_{i j}}^{0} f_{i j}(\xi) x_{j}(s+\xi) d \xi \\
& \left.\left.+\sum_{j=1}^{n} c_{i j}(s) \int_{-\sigma_{i j}}^{0} g_{i j}(\xi) x_{j}^{\prime}(s+\xi) d \xi\right] d s\right\} \\
& =\left[G_{i}(t, t+\omega) x_{i}(t+\omega)\right. \\
& \times\left(\sum_{j=1}^{n} a_{i j}(t+\omega) x_{j}(t+\omega)\right. \\
& +\sum_{j=1}^{n} b_{i j}(t+\omega) \int_{-\tau_{i j}}^{0} f_{i j}(\xi) x_{j}(t+\omega+\xi) d \xi \\
& \left.+\sum_{j=1}^{n} c_{i j}(t+\omega) \int_{-\sigma_{i j}}^{0} g_{i j}(\xi) x_{j}^{\prime}(t+\omega+\xi) d \xi\right) \\
& -G_{i}(t, t) x_{i}(t) \\
& \times\left(\sum_{j=1}^{n} a_{i j}(t) x_{j}(t)+\sum_{j=1}^{n} b_{i j}(t)\right. \\
& \times \int_{-\tau_{i j}}^{0} f_{i j}(\xi) x_{j}(t+\xi) d \xi \\
& \left.\left.+\sum_{j=1}^{n} c_{i j}(t) \int_{-\sigma_{i j}}^{0} g_{i j}(\xi) x_{j}^{\prime}(t+\xi) d \xi\right)\right] \\
& +r_{i}(t) x_{i}(t) \\
& =x_{i}(t)\left[r_{i}(t)-\sum_{j=1}^{n} a_{i j}(t) x_{j}(t)\right. \\
& -\sum_{j=1}^{n} b_{i j}(t) \int_{-\tau_{i j}}^{0} f_{i j}(\xi) x_{j}(t-\xi) d \xi \\
& \left.-\sum_{j=1}^{n} c_{i j}(t) \int_{-\sigma_{i j}}^{0} g_{i j}(\xi) x_{j}^{\prime}(t+\xi) d \xi\right] .
\end{aligned}
$$

For any $t=t_{j}, j \in Z_{+}$, we have from (6) that

$$
\begin{aligned}
& x_{i}\left(t_{j}^{+}\right)-x_{i}\left(t_{j}\right) \\
& =\int_{t_{j}}^{t_{j}+\omega}\left[G_{i}\left(t_{j}^{+}, s\right)-G_{i}\left(t_{j}, s\right)\right] x_{i}(s) \\
& \quad \times\left[\sum_{j=1}^{n} a_{i j}(s) x_{j}(s)+\sum_{j=1}^{n} b_{i j}(s) \int_{-\tau_{i j}}^{0} f_{i j}(\xi) x_{j}(s+\xi) d \xi\right. \\
& \left.\quad+\sum_{j=1}^{n} c_{i j}(s) \int_{-\sigma j}^{0} g_{i j}(\xi) x_{j}^{\prime}(s+\xi) d \xi\right] d s \\
& \quad+\sum_{t_{j}^{+} \leq t_{k}<t_{j}+\omega} G_{i}\left(t_{j}^{+}, t_{k}\right) I_{i k}\left(x_{i}\left(t_{k}\right)\right) \\
& \quad-\sum_{t_{j} \leq t_{k}<t_{j}+\omega} G_{i}\left(t_{j}, t_{k}\right) I_{i k}\left(x_{i}\left(t_{k}\right)\right) \\
& =-I_{i k}\left(x\left(t_{k}\right)\right) .
\end{aligned}
$$

Hence $x_{i}(t)$ is a positive $\omega$-periodic solution of (2). Thus we complete the proof of Lemma 7.

Lemma 8 (see $[20,23])$. Let $E$ be a cone of the real Banach space $X$ and $E_{r, R}=\{x \in E: r \leq\|x\| \leq R\}$ with $0<r<R$. Assume that $A: E_{r, R} \rightarrow E$ is strict-set-contractive such that one of the following two conditions is satisfied:

(a) Ax $\npreceq x$, for all $x \in E,\|x\|=r$ and $A x \notin$ $x$, for all $x \in E,\|x\|=R$;

(b) $A x \notin x$, for all $x \in E,\|x\|=r$ and $A x \notin$ $x$, for all $x \in E,\|x\|=R$.

Then $A$ has at least one fixed point in $E_{r, R}$. In order to apply Lemma 7 to system (1), one sets

$$
\begin{gathered}
P C(R)=\left\{x=\left(x_{1}, x_{2}, \ldots, x_{n}\right)^{T}:\right. \\
R \longrightarrow R \mid x \in C\left(\left(t_{k}, t_{k+1}\right), R\right), \\
\left.\exists x\left(t_{k}^{-}\right)=x\left(t_{k}\right), x\left(t_{k}^{+}\right), k \in N\right\}, \\
P C^{1}(R)=\left\{x=\left(x_{1}, x_{2}, \cdots, x_{n}\right)^{T}:\right. \\
R \longrightarrow R \mid x \in C^{1}\left(\left(t_{k}, t_{k+1}\right), R\right), \\
\left.\exists x^{\prime}\left(t_{k}^{-}\right)=x^{\prime}\left(t_{k}\right), x\left(t_{k}^{+}\right), k \in N, t \in R\right\} .
\end{gathered}
$$

Define

$$
X=\left\{x=\left(x_{1}, x_{2}, \ldots, x_{n}\right)^{T}: x \in P C(R) \mid x(t+\omega)=x(t)\right\}
$$


with the norm defined by $\|x\|_{0}=\sum_{i=1}^{n}\left|x_{i}\right|_{0}$, where $\left|x_{i}\right|_{0}=$ $\sup _{t \in[0, \omega]}\left\{\left|x_{i}(t)\right|\right\}, i=1,2, \ldots, n$, and

$$
\begin{aligned}
Y=\{x & =\left(x_{1}, x_{2}, \ldots, x_{n}\right)^{T}: x \in P C^{1}(R) \mid x(t+\omega) \\
& =x(t), t \in R\}
\end{aligned}
$$

with the norm defined by $\|x\|_{1}=\sum_{i=1}^{n}\left|x_{i}\right|_{1}$, where $\left|x_{i}\right|_{1}=$ $\max \left\{\left|x_{i}\right|_{0},\left|x_{i}^{\prime}\right|_{0}\right\}$. Then $X$ and $Y$ are both Banach spaces. Define the cone $E$ in $Y$ by

$$
\begin{gathered}
E=\left\{x=\left(x_{1}, x_{2}, \ldots, x_{n}\right)^{T}:\left.x \in P C^{1}(R)\left|x_{i}(t)=\delta_{j}\right| x_{i}(t)\right|_{1},\right. \\
t \in[0, \omega], i, j=1,2 \ldots, n\} .
\end{gathered}
$$

Let the map $T$ be defined by

$$
(T x)(t)=\left(\left(T_{1} x\right)(t),\left(T_{2} x\right)(t), \ldots,\left(T_{n} x\right)(t)\right)^{T},
$$

where $x \in E, t \in R$,

$$
\begin{aligned}
& \left(T_{i} x\right)(t) \\
& =\int_{t}^{t+\omega} G_{i}(t, s) x_{i}(s) \\
& \quad \times\left[\sum_{j=1}^{n} a_{i j}(s) x_{j}(s)\right. \\
& \quad+\sum_{j=1}^{n} b_{i j}(s) \int_{-\tau_{i j}}^{0} f_{i j}(\xi) x_{j}(s+\xi) d \xi \\
& \left.\quad+\sum_{j=1}^{n} c_{i j}(s) \int_{-\sigma_{i j}}^{0} g_{i j}(\xi) x_{j}^{\prime}(s+\xi) d \xi\right] d s \\
& +\sum_{t \leq t_{k}<t+\omega} G_{i}\left(t, t_{k}\right) I_{i k}\left(x_{i}\left(t_{k}\right)\right),
\end{aligned}
$$

where

$$
G_{i}(t, s)=\frac{e^{-\int_{t}^{s} r_{i}(\theta) d \theta}}{1-e^{-\int_{0}^{\omega} r_{i}(\theta) d \theta}}, \quad s \in[t, t+\omega], i=1,2, \ldots, n .
$$

It is obvious to see that $G_{i}(t+\omega, s+\omega)=G_{i}(t, s), \partial G_{i}(t, s) / \partial t=$ $r_{i}(t) G_{i}(t, s), G_{i}(t, t+\omega)-G_{i}(t, t)=-1$, and

$$
\frac{\delta_{i}}{1-\delta_{i}} \leq G_{i}(t, s) \leq \frac{1}{1-\delta_{i}}, \quad s \in[t, t+\omega], i=1,2, \ldots, n .
$$

In what follows, we will give some lemmas concerning $E$ and $A$ defined by (17) and (18), respectively.

Lemma 9. Assume that $\left(A_{1}\right)-\left(A_{3}\right)$ hold.

(i) If $\max \left\{r_{i}^{M}, i=1,2, \ldots, n\right\} \leq 1$, then $T: E \rightarrow E$ is well defined. (ii) If $\left(A_{4}\right)$ holds and $\max \left\{r_{i}^{M}, i=1,2, \ldots, n\right\}>1$, then $T: E \rightarrow E$ is well defined.

Proof. For any $x \in E$, it is clear that $T x \in P C^{1}(R)$. From (18), for $t \in[0, \omega]$, we have

$$
\begin{aligned}
& \left(T_{i} x\right)(t+\omega) \\
& =\int_{t+\omega}^{t+2 \omega} G_{i}(t+\omega, s) x_{i}(s) \\
& \times\left[\sum_{j=1}^{n} a_{i j}(s) x_{j}(s)+\sum_{j=1}^{n} b_{i j}(s) \int_{-\tau_{i j}}^{0} f_{i j}(\xi) x_{j}(s+\xi) d \xi\right. \\
& \left.+\sum_{j=1}^{n} c_{i j}(s) \int_{-\sigma_{i j}}^{0} g_{i j}(\xi) x_{j}^{\prime}(s+\xi) d \xi\right] d s \\
& +\sum_{t+\omega \leq t_{k}<t+2 \omega} G_{i}\left(t+\omega, t_{k}\right) I_{i k}\left(x_{i}\left(t_{k}\right)\right) \\
& =\int_{t}^{t+\omega} G_{i}(t+\omega, u+\omega) x_{i}(u+\omega) \\
& \times\left[\sum_{j=1}^{n} a_{i j}(u+\omega) x_{j}(u+\omega)+\sum_{j=1}^{n} b_{i j}(u+\omega)\right. \\
& \times \int_{-\tau_{i j}}^{0} f_{i j}(\xi) x_{j}(u+\omega+\xi) d \xi \\
& \left.+\sum_{j=1}^{n} c_{i j}(u+\omega) \int_{-\sigma_{i j}}^{0} g_{i j}(\xi) x_{j}^{\prime}(u+\omega+\xi) d \xi\right] d s \\
& +\sum_{t \leq t_{k}<t+\omega} G_{i}\left(t, t_{k}\right) I_{i k}\left(x_{i}\left(t_{k}\right)\right) \\
& =\int_{t}^{t+\omega} G_{i}(t, u) x_{i}(u) \\
& \times\left[\sum_{j=1}^{n} a_{i j}(u) x_{j}(u)+\sum_{j=1}^{n} b_{i j}(u) \int_{-\tau_{i j}}^{0} f_{i j}(\xi) x_{j}(u+\xi) d \xi\right. \\
& \left.+\sum_{j=1}^{n} c_{i j}(u) \int_{-\sigma_{i j}}^{0} g_{i j}(\xi) x_{j}^{\prime}(u+\xi) d \xi\right] d s \\
& +\sum_{t \leq t_{k}<t+\omega} G_{i}\left(t, t_{k}\right) I_{i k}\left(x_{i}\left(t_{k}\right)\right) \\
& =\left(T_{i} x\right)(t), \quad i=1,2, \ldots, n .
\end{aligned}
$$

That is, $\left(T_{i} x\right)(t+\omega)=\left(T_{i} x\right)(t), t \in[0, \omega]$. So $T x \in Y$. In view of $\left(A_{2}\right)$, for $x \in E, t \in[0, \omega]$, we have 
6

International Journal of Differential Equations

$$
\begin{aligned}
& \sum_{j=1}^{n} a_{i j}(t) x_{j}(t)+\sum_{j=1}^{n} b_{i j}(t) \int_{-\tau_{i j}}^{0} f_{i j}(\xi) x_{j}(t+\xi) d \xi \\
& \quad+\sum_{j=1}^{n} c_{i j}(t) \int_{-\sigma_{i j}}^{0} g_{i j}(\xi) x_{j}^{\prime}(t+\xi) d \xi \\
& \quad \geq \sum_{j=1}^{n} a_{i j}(t) \delta_{j}\left|x_{j}\right|_{1}-\sum_{j=1}^{n} b_{i j}(t)\left|x_{j}\right|_{1}-\sum_{j=1}^{n} c_{i j}(t)\left|x_{j}\right|_{1} \\
& \quad=\sum_{j=1}^{n}\left[a_{i j}(t) \delta_{j}-b_{i j}(t)-c_{i j}(t)\right]\left|x_{j}^{\prime}\right|_{1} \geq 0, \quad i=1,2, \ldots, n .
\end{aligned}
$$

Therefore, for $x \in E, t \in[0, \omega]$, we find

$$
\begin{aligned}
& \left|T_{i} x\right|_{0} \\
& \leq \frac{1}{1-\delta_{i}}\left\{\int_{0}^{\omega} x_{i}(s)\right. \\
& \times\left[\sum_{j=1}^{n} a_{i j}(s) x_{j}(s)+\sum_{j=1}^{n} b_{i j}(s)\right. \\
& \times \int_{-\tau_{i j}}^{0} f_{i j}(\xi) x_{j}(s+\xi) d \xi \\
& \left.+\sum_{j=1}^{n} c_{i j}(s) \int_{-\sigma_{i j}}^{0} g_{i j}(\xi) x_{j}^{\prime}(s+\xi) d \xi\right] d s \\
& \left.+\sum_{t \leq t_{k}<t+\omega} I_{i k}\left(x_{i}\left(t_{k}\right)\right)\right\}, \\
& \left(T_{i} x\right)(t) \\
& \geq \frac{\delta_{i}}{1-\delta_{i}}\left\{\int_{t}^{t+\omega} x_{i}(s)\right. \\
& \times\left[\sum_{j=1}^{n} a_{i j}(s) x_{j}(s)\right. \\
& +\sum_{j=1}^{n} b_{i j}(s) \int_{-\tau_{i j}}^{0} f_{i j}(\xi) x_{j}(s+\xi) d \xi \\
& \left.+\sum_{j=1}^{n} c_{i j}(s) \int_{-\sigma_{i j}}^{0} g_{i j}(\xi) x_{j}^{\prime}(s+\xi) d \xi\right] d s \\
& \left.+\sum_{t \leq t_{k}<t+\omega} I_{i k}\left(x_{i}\left(t_{k}\right)\right)\right\}
\end{aligned}
$$

$$
\begin{gathered}
=\frac{\delta_{i}}{1-\delta_{i}} \\
\times\left\{\int_{0}^{\omega} x_{i}(s)\right. \\
\times\left[\sum_{j=1}^{n} a_{i j}(s) x_{j}(s)\right. \\
+\sum_{j=1}^{n} b_{i j}(s) \int_{-\tau_{i j}}^{0} f_{i j}(\xi) x_{j}(s+\xi) d \xi \\
\left.+\sum_{j=1}^{n} c_{i j}(s) \int_{-\sigma_{i j}}^{0} g_{i j}(\xi) x_{j}^{\prime}(s+\xi) d \xi\right] d s \\
\left.+\sum_{t \leq t_{k}<t+\omega} I_{i k}\left(x_{i}\left(t_{k}\right)\right)\right\} \\
\geq \delta_{i}\left|T_{i} x\right|_{0}, \quad i=1,2, \ldots, n .
\end{gathered}
$$

Now, we show that $\left(T_{i} x\right)(t) \geq \delta_{i}\left|T_{i} x\right|_{1}, i=1,2, \ldots, n, t \in$ $[0, \omega]$. From (18), we obtain

$$
\begin{aligned}
& \left(T_{i} x\right)^{\prime}(t) \\
& =G_{i}(t, t+\omega) x_{i}(t+\omega) \\
& \times\left[\sum_{j=1}^{n} a_{i j}(t+\omega) x_{j}(t+\omega)\right. \\
& +\sum_{j=1}^{n} b_{i j}(t+\omega) \int_{-\tau_{i j}}^{0} f_{i j}(\xi) x_{j}(t+\omega+\xi) d \xi \\
& \left.+\sum_{j=1}^{n} c_{i j}(t+\omega) \int_{-\sigma_{i j}}^{0} g_{i j}(\xi) x_{j}^{\prime}(t+\omega+\xi) d \xi\right] \\
& -G_{i}(t, t) x_{i}(t) \\
& +\sum_{i}(t)\left(T_{i} x\right)(t) \\
& +r_{i}(t)\left(T_{i} x\right)(t)-x_{i}(t) x_{j}(t)+\sum_{j=1}^{n} b_{i j}(t) \\
& \times \int_{-\tau_{i j}}^{0} f_{i j}(\xi) x_{j}(t+\xi) d \xi \\
& \left.g_{-\sigma_{i j}} g_{i j}(\xi) x_{j}^{\prime}(t+\xi) d \xi\right]
\end{aligned}
$$




$$
\begin{gathered}
\times\left[\sum_{j=1}^{n} a_{i j}(s) x_{j}(s)+\sum_{j=1}^{n} b_{i j}(t) \int_{-\tau_{i j}}^{0} f_{i j}(\xi) x_{j}(t+\xi) d \xi\right. \\
\left.+\sum_{j=1}^{n} c_{i j}(t) \int_{-\sigma_{i j}}^{0} g_{i j}(\xi) x_{j}^{\prime}(t+\xi) d \xi\right], \\
i=1,2, \ldots, n .
\end{gathered}
$$

It follows from (23) and (25) that if $\left(T_{i} x\right)^{\prime}(t) \geq 0, i=$ $1,2, \ldots, n$, then

$$
\begin{array}{r}
\left(T_{i} x\right)^{\prime}(t) \leq r_{i}(t)\left(T_{i} x\right)(t) \leq r_{i}^{M}\left(T_{i} x\right)(t) \leq\left(T_{i} x\right)(t), \\
i=1,2, \ldots, n .
\end{array}
$$

On the other hand, from (25) and $\left(A_{3}\right)$, if $\left(T_{i} x\right)^{\prime}(t)<0, i=$ $1,2, \ldots, n$, then

$$
\begin{aligned}
- & \left(T_{i} x\right)^{\prime}(t) \\
= & -r_{i}(t)\left(T_{i} x\right)(t)+x_{i}(t) \\
& \times\left[\sum_{j=1}^{n} a_{i j}(t) x_{j}(t)+\sum_{j=1}^{n} b_{i j}(t) \int_{-\tau_{i j}}^{0} f_{i j}(\xi) x_{j}(t+\xi) d \xi\right. \\
& \left.+\sum_{j=1}^{n} c_{i j}(t) \int_{-\sigma_{i j}}^{0} g_{i j}(\xi) x_{j}^{\prime}(t+\xi) d \xi\right] \\
\leq & \left|x_{i}\right|_{1} \sum_{j=1}^{n}\left[a_{i j}(t)+b_{i j}(t)+c_{i j}(t)\right]\left|x_{i}\right|_{1}-r_{i}^{L}\left(T_{i} x\right)(t) \\
\leq & \left(1+r_{i}^{L}\right) \frac{\delta_{i}^{2}}{1-\delta_{i}}\left|x_{i}\right|_{1} \\
& \times \sum_{j=1}^{n}\left\{\int_{0}^{\omega}\left[\delta_{j} a_{i j}(s)-b_{i j}(s)-c_{i j}(s)\right]\left|x_{i}\right|_{1} d s\right\} \\
& -r_{i}^{L}\left(T_{i} x\right)(t) \\
= & \left(1+r_{i}^{L}\right) \int_{t}^{t+\omega} \frac{\delta_{i}}{1-\delta_{i}} \delta_{i}\left|x_{i}\right|_{1} \\
\leq & \times \sum_{j=1}^{n}\left[\delta_{j} a_{i j}(s)-b_{i j}(s)-c_{i j}(s)\right]\left|x_{i}\right|_{1} d s-r_{i}^{L}\left(T_{i} x\right)(t) \\
& \times \sum_{j=1}^{L} a_{i j}(s) x_{j}(s)+\sum_{j=1}^{n} b_{i j}(s) \\
t+\omega & f_{i j}(t, s) x_{i}(s) \\
& \\
& \\
&
\end{aligned}
$$

$$
\begin{gathered}
\left.+\sum_{j=1}^{n} c_{i j}(s) \int_{-\sigma_{i j}}^{0} g_{i j}(\xi) x_{j}^{\prime}(s+\xi) d \xi\right] d s \\
\left.+\sum_{t \leq t_{k}<t+\omega} G_{i}\left(t, t_{k}\right) I_{i k}\left(x_{i}\left(t_{k}\right)\right)\right\}-r_{i}^{L}\left(T_{i} x\right)(t) \\
=\left(1+r_{i}^{L}\right)\left(T_{i} x\right)(t)-r_{i}^{L}\left(T_{i} x\right)(t) \\
=\left(T_{i} x\right)(t), \quad i=1,2, \ldots, n .
\end{gathered}
$$

It follows from (27) and (28) that $\left|\left(T_{i} x\right)^{\prime}\right|_{0} \leq\left|T_{i} x\right|_{0}, i=$ $1,2, \ldots, n$. So $\left|T_{i} x\right|_{1}=\left|T_{i} x\right|_{0}, i=1,2, \ldots, n$. By (25), we have $\left(T_{i} x\right)(t) \geq \delta_{i}\left|T_{i} x\right|_{1}, i=1,2, \ldots, n$. Hence, $T x \in E$. This completes the proof of (i). In view of the proof of (ii), we only need to prove that $\left(T_{i} x\right)^{\prime}(t) \geq 0, i=1,2, \ldots, n$, implies

$$
\left(T_{i} x\right)^{\prime}(t) \leq\left(T_{i} x\right)(t), \quad i=1,2, \ldots, n .
$$

From (23), (26), $\left(A_{2}\right)$, and $\left(A_{4}\right)$, we have

$$
\begin{aligned}
& \left(T_{i} x\right)^{\prime}(t) \\
& =r_{i}(t)\left(T_{i} x\right)(t)-x_{i}(t) \\
& \quad \times\left[\sum_{j=1}^{n} a_{i j}(t) x_{j}(t)-\sum_{j=1}^{n} b_{i j}(t) \int_{-\tau_{i j}}^{0} f_{i j}(\xi) x_{j}(t+\xi) d \xi\right. \\
& \left.\quad-\sum_{j=1}^{n} c_{i j}(t) \int_{-\sigma_{i j}}^{0} g_{i j}(\xi) x_{j}^{\prime}(t+\xi) d \xi\right] \\
& \leq r_{i}(t)\left(T_{i} x\right)(t)-\delta_{i}\left|x_{i}\right|_{1} \\
& \quad \times \sum_{j=1}^{n}\left[\delta_{j} a_{i j}(t)-b_{i j}(t)-c_{i j}(t)\right]\left|x_{j}\right|_{1} \\
& \leq r_{i}^{M}\left(T_{i} x\right)(t)-\delta_{i}\left|x_{i}\right|_{1} \frac{r_{i}^{M}-1}{\delta_{i}\left(1-\delta_{i}\right)} \\
& \quad \times \sum_{j=1}^{n}\left\{\int_{0}^{\omega}\left[a_{i j}(s)+b_{i j}(s)+c_{i j}(s)\right]\left|x_{j}\right|_{1} d s\right\} \\
& =r_{i}^{M}\left(T_{i} x\right)(t)-\left(r_{i}^{M}-1\right) \int_{t}^{t+\omega} \frac{1}{1-\delta_{i}}\left|x_{i}\right|_{1} \\
& \quad \times \sum_{j=1}^{n}\left[a_{i j}(s)+b_{i j}(s)+c_{i j}(s)\right]\left|x_{j}\right|_{1} d s \\
&
\end{aligned}
$$




$$
\begin{gathered}
\times\left\{\int_{t}^{t+\omega} G_{i}(t, s) x_{i}(s)\right. \\
\times\left[\sum_{j=1}^{n} a_{i j}(s) x_{j}(s)+\sum_{j=1}^{n} b_{i j}(s)\right. \\
\quad \times \int_{-\tau_{i j}}^{0} f_{i j}(\xi) x_{j}(s+\xi) d \xi \\
\left.\quad+\sum_{j=1}^{n} c_{i j}(s) \int_{-\sigma_{i j}}^{0} g_{i j}(\xi) x_{j}^{\prime}(s+\xi) d \xi\right] d s \\
\left.+\sum_{t \leq t_{k}<t+\omega} G_{i}\left(t, t_{k}\right) I_{i k}\left(x_{i}\left(t_{k}\right)\right)\right\} \\
=r_{i}^{M}\left(T_{i} x\right)(t)-\left(r_{i}^{M}-1\right)\left(T_{i} x\right)(t)=\left(T_{i} x\right)(t), \\
i=1,2, \ldots, n .
\end{gathered}
$$

The proof of (ii) is complete. Thus we complete the proof of Lemma 9.

Lemma 10. Assume that $\left(A_{1}\right)-\left(A_{3}\right)$ hold and $R \max _{1 \leq j \leq n}\left\{\sum_{j=1}^{n} b_{i j}^{M}\right\}<1$.

(i) If $\max \left\{r_{i}^{M}, i=1,2, \ldots, n\right\} \leq 1$, then $T: E \bigcap \overline{\Omega_{R}} \rightarrow E$ is strict-set-contractive.

(ii) If $\left(A_{4}\right)$ holds and $\min \left\{r_{i}^{M}, i=1,2, \ldots, n\right\}>1$, then $T: E \cap \overline{\Omega_{R}} \rightarrow E$ is strict-set-contractive, where $\Omega_{R}=$ $\left\{x \in Y:|x|_{1}<R\right\}$.

Proof. We only need to prove (i), since the proof of (ii) is similar. It is easy to see that $T$ is continuous and bounded. Now we prove that a $\alpha_{Y}(T(S)) \leq R \max _{1 \leq j \leq n}\left\{\sum_{j=1}^{n} b_{i j}^{M}\right\} \alpha_{Y}(S)$ for any bounded set $S \in \bar{\Omega}_{R}$. Let $\eta=\alpha_{Y}(S)$. Then, for any positive number $\epsilon<R \max _{1 \leq j \leq n}\left\{\sum_{j=1}^{n} b_{i j}^{M}\right\} \eta$, there is a finite family of subsets $\left\{S_{i}\right\}$ satisfying $S=\bigcup_{i} S_{i}$ with $\operatorname{diam}\left(S_{i}\right) \leq \eta+\epsilon$. Therefore

$$
|x-y|_{1} \leq \eta+\epsilon, \quad \text { for any } x, y \in S_{i} .
$$

As $S$ and $S_{i}$ are precompact in $X$, it follows that there is a finite family of subsets $\left\{S_{i j}\right\}$ of $S_{i}$ such that $S_{i}=\bigcup_{j} S_{i j}$ and

$$
|x-y|_{0} \leq \epsilon, \quad \text { for any } x, y \in S_{i j} .
$$

In addition, for any $x \in S$ and $t \in[0, \omega]$, we have

$$
\begin{aligned}
& \left(T_{i} x\right)(t) \\
& =\int_{t}^{t+\omega} G_{i}(t, s) x_{i}(s) \\
& \quad \times\left[\sum_{j=1}^{n} a_{i j}(s) x_{j}(s)+\sum_{j=1}^{n} b_{i j}(s) \int_{-\tau_{i j}}^{0} f_{i j}(\xi) x_{j}(s+\xi) d \xi\right. \\
& \left.\quad+\sum_{j=1}^{n} c_{i j}(s) \int_{-\sigma_{i j}}^{0} g_{i j}(\xi) x_{j}^{\prime}(s+\xi) d \xi\right] d s \\
& \quad+\sum_{t \leq t_{k}<t+\omega} G_{i}\left(t, t_{k}\right) I_{i k}\left(x_{i}\left(t_{k}\right)\right) \\
& \leq \frac{R^{2}}{1-\delta_{i}} \int_{0}^{\omega}\left[\sum_{j=1}^{n} a_{i j}(s)+\sum_{j=1}^{n} b_{i j}(s)+\sum_{j=1}^{n} c_{i j}(s)\right] d s \\
& \quad+\frac{1}{1-\delta_{i}} \sum_{t \leq t_{k}<t+\omega} I_{i k}\left(x_{i}\left(t_{k}\right)\right):=B_{i}, \quad i=1,2, \ldots, n,
\end{aligned}
$$

$$
\left|\left(T_{i} x\right)^{\prime}(t)\right|
$$

$$
\begin{aligned}
& =\mid r_{i}(t)\left(T_{i} x\right)(t)-x_{i}(t) \\
& \quad \times\left[\sum_{j=1}^{n} a_{i j}(t) x_{j}(t)+\sum_{j=1}^{n} b_{i j}(t) \int_{-\tau_{i j}}^{0} f_{i j}(\xi) x_{j}(t+\xi) d \xi\right. \\
& \left.\quad+\sum_{j=1}^{n} c_{i j}(t) \int_{-\sigma_{i j}}^{0} g_{i j}(\xi) x_{j}^{\prime}(t+\xi) d \xi\right] \mid \\
& \leq r_{i}^{M} B_{i}+R^{2} \sum_{j=1}^{n}\left(a_{i j}^{M}+b_{i j}^{M}+c_{i j}^{M}\right), \quad i=1,2, \ldots, n .
\end{aligned}
$$

Hence,

$$
\begin{gathered}
\|(T x)\|_{0} \leq \sum_{i=1}^{n} B_{i}, \\
\left\|(T x)^{\prime}\right\|_{0} \leq \sum_{i=1}^{n}\left[B_{i} r_{i}^{M} B_{i}+R^{2} \sum_{j=1}^{n}\left(a_{i j}^{M}+b_{i j}^{M}\right)\right] .
\end{gathered}
$$

Applying the Arzela-Ascoli theorem, we know that $T(S)$ is precompact in $X$. Then, there is a finite family of subsets $\left\{S_{i j k}\right\}$ of $S_{i j}$ such that $S_{i j}=\bigcup_{k} S_{i j k}$ and

$$
|T x-T y|_{0} \leq \epsilon, \quad \text { for any } x, y \in S_{i j k} .
$$


From (23), (26), (31)-(34), and $\left(A_{2}\right)$, for any $x, y \in S_{i j k}$, we have

$$
\begin{gathered}
\left|\left(T_{i} x\right)^{\prime}-\left(T_{i} y\right)^{\prime}\right|_{0} \\
=\max _{t \in[0, \omega]}\left\{\mid r_{i}(t)\left(T_{i} x\right)(t)-r_{i}(t)\left(T_{i} y\right)(t)-x_{i}(t)\right. \\
\times\left[\sum_{j=1}^{n} a_{i j}(t) x_{j}(t)\right. \\
+\sum_{j=1}^{n} b_{i j}(t) \int_{-\tau_{i j}}^{0} f_{i j}(\xi) x_{j}(t+\xi) d \xi \\
\left.+\sum_{j=1}^{n} c_{i j}(t) \int_{-\sigma_{i j}}^{0} g_{i j}(\xi) x_{j}^{\prime}(t+\xi) d \xi\right] \\
+y_{i}(t)\left[\sum_{j=1}^{n} a_{i j}(t) y_{j}(t)\right. \\
+\sum_{j=1}^{n} b_{i j}(t) \int_{-\tau_{i j}}^{0} f_{i j}(\xi) y_{j}(t+\xi) d \xi \\
\left.\left.+\sum_{j=1}^{n} c_{i j}(t) \int_{-\sigma_{i j}}^{0} g_{i j}(\xi) y_{j}^{\prime}(t+\xi) d \xi\right] \mid\right\}
\end{gathered}
$$$$
\leq \max _{t \in[0, \omega]}\left\{\left|r_{i}(t)\left[\left(T_{i} x\right)(t)-\left(T_{i} y\right)(t)\right]\right|\right\}
$$$$
+\max _{t \in[0, \omega]}\left\{\mid x_{i}\left[\left(\sum_{j=1}^{n} a_{i j}(t) x_{j}(t)\right.\right.\right.
$$$$
+\sum_{j=1}^{n} b_{i j}(t) \int_{-\tau_{i j}}^{0} f_{i j}(\xi) x_{j}(t+\xi) d \xi
$$$$
\left.+\sum_{j=1}^{n} c_{i j}(t) \int_{-\sigma_{i j}}^{0} g_{i j}(\xi) x_{j}^{\prime}(t+\xi) d \xi\right)
$$$$
-\left(\sum_{j=1}^{n} a_{i j}(t) y_{j}(t)+\sum_{j=1}^{n} b_{i j}(t)\right.
$$$$
\times \int_{-\tau_{i j}}^{0} f_{i j}(\xi) y_{j}(t+\xi) d \xi+\sum_{j=1}^{n} c_{i j}(t)
$$$$
\left.\left.\left.\times \int_{-\sigma_{i j}}^{0} g_{i j}(\xi) y_{j}^{\prime}(t+\xi) d \xi\right)\right] \mid\right\}
$$$$
+\max _{t \in[0, \omega]}\left\{\mid\left[x_{i}(t)-y_{i}(t)\right]\right.
$$

$$
\begin{aligned}
& \times\left(\sum_{j=1}^{n} a_{i j}(t) y_{j}(t)+\sum_{j=1}^{n} b_{i j}(t)\right. \\
& \quad \times \int_{-\tau_{i j}}^{0} f_{i j}(\xi) y_{j}(t+\xi) d \xi \\
& \left.\left.\quad+\sum_{j=1}^{n} c_{i j}(t) \int_{-\sigma_{i j}}^{0} g_{i j}(\xi) y_{j}^{\prime}(t+\xi) d \xi\right) \mid\right\}
\end{aligned}
$$$$
\leq r_{i}^{M}\left|\left(T_{i} x\right)-\left(T_{i} y\right)\right|_{0}
$$$$
+R \max _{t \in[0, \omega]}\left\{\sum _ { j = 1 } ^ { n } \left[a_{i j}(t)\left|x_{j}(t)-y_{j}(t)\right|+b_{i j}(t)\right.\right.
$$$$
\times \int_{-\tau_{i j}}^{0} f_{i j}(\xi)
$$$$
\times\left|x_{i}(t+\xi)-y_{i}(t+\xi)\right| d \xi+c_{i j}(t)
$$$$
\times \int_{-\sigma_{i j}}^{0} g_{i j}(\xi)
$$$$
\left.\left.\times\left|x_{j}^{\prime}(t+\xi)-y^{\prime}(t-\xi)\right| d \xi\right]\right\}
$$$$
+\max _{t \in[0, \omega]}\left\{\mid\left[x_{i}(t)-y_{i}(t)\right]\right.
$$$$
\times\left(\sum_{j=1}^{n} a_{i j}(t) y_{j}(t)\right.
$$$$
+\sum_{j=1}^{n} b_{i j}(t) \int_{-\tau_{i j}}^{0} f_{i j}(\xi) y_{j}(t+\xi) d \xi
$$$$
+\sum_{j=1}^{n} c_{i j}(t)
$$$$
\left.\left.\times \int_{-\sigma_{i j}}^{0} g_{i j}(\xi) y_{j}^{\prime}(t+\xi) d \xi\right) \mid\right\}
$$

$$
\leq r_{i}^{M} \epsilon+R \epsilon \sum_{j=1}^{n} a_{i j}^{M}+R \epsilon \sum_{j=1}^{n} b_{i j}^{M}+\left|x_{i}\right|_{0}(\eta+\epsilon)
$$$$
\times \sum_{j=1}^{n} c_{i j}^{M}+R \epsilon \sum_{j=1}^{n}\left(a_{i j}^{M}+b_{i j}^{M}+c_{i j}^{M}\right)
$$$$
=\left|x_{i}\right|_{0} \sum_{j=1}^{n} c_{i j}^{M} \eta+\widetilde{B}_{i} \epsilon
$$

where

$$
\widetilde{B}_{i}=r_{i}^{M}+2 R \sum_{j=1}^{n}\left(a_{i j}^{M}+b_{i j}^{M}+c_{i j}^{M}\right), \quad i=1,2, \ldots, n .
$$


From (36) and (37) we obtain

$$
\begin{aligned}
& \|T x-T y\|_{1} \leq\left(\sum_{i=1}^{n}\left|x_{i}\right|_{0} \sum_{j=1}^{n} c_{i j}^{M}\right) \eta+\epsilon \sum_{i=1}^{n} \widetilde{B}_{i} \\
& \leq R \max _{1 \leq i \leq n}\left\{\sum_{j=1}^{n} c_{i j}^{M}\right\} \eta+\epsilon \sum_{i=1}^{n} \widetilde{B}_{i}, \quad \text { for any } x, y \in S_{i j k} .
\end{aligned}
$$

As $\epsilon$ is arbitrarily small, it follows that

$$
\alpha_{Y}(T(S)) \leq R \max _{1 \leq j \leq n}\left\{\sum_{j=1}^{n} c_{i j}^{M}\right\} \alpha_{Y}(S)
$$

Therefore, $T$ is strict-set-contractive. The proof of Lemma 10 is complete.

\section{Main Results}

Our main result of this paper is as follows.

Theorem 11. Assume that $\left(A_{1}\right)-\left(A_{3}\right),\left(A_{5}\right)$ hold.

(i) If $\max \left\{r_{i}^{M}, i=1,2, \ldots, n\right\} \leq 1$, then system (2) has at least one positive $\omega$-periodic solution.

(ii) If $\left(A_{4}\right)$ holds and $\min \left\{r_{i}^{M}, i=1,2, \ldots, n\right\}>1$, then system (2) has at least one positive $\omega$-periodic solution.

Proof. We only need to prove (i), since the proof of (ii) is similar. Let

$$
\begin{aligned}
& R=\left(\min _{i \in[1, n]}\left\{\frac{\delta_{i}^{2}}{1-\delta_{i}} \min _{j \in[1, n]}\left\{\alpha_{i j}\right\}\right\}\right)^{-1}, \\
& 0<r<\min _{i \in[1, n]}\left\{\frac{\delta_{i}\left(1-\delta_{i}\right)-\gamma_{i}}{\max _{j \in[1, n]}\left\{\beta_{i j}\right\}}\right\} .
\end{aligned}
$$

Then it is easy to see that $0<r<R$. From Lemmas 9 and 10 , we know that $T$ is strict-set-contractive on $E_{r, R}$. In view of (26), we see that if there exists $x^{*} \in E$ such that $T x^{*}=$ $x^{*}$, then $x^{*}$ is one positive $\omega$-periodic solution of system (2). Now, we will prove that condition (ii) of Lemma 7 holds. First, we prove that $T x \geq x$, for all $x \in E,\|x\|_{1}<r$. Otherwise, there exists $x \in E,\|x\|_{1}<r$, such that $T x \geq x$. So $\|x\|_{0}>0$ and $T x-x \geq 0$, which implies that

$$
\begin{aligned}
& T_{i} x(t)-x_{i}(t) \geq \delta_{i}\left|T_{i} x-x_{i}\right|_{1} \geq 0, \\
& \text { for any } t \in[0, \omega], i=1,2, \ldots, n
\end{aligned}
$$

Moreover, for $t \in[0, \omega]$, we have

$$
\begin{aligned}
& \left(T_{i} x\right)(t) \\
& =\int_{t}^{t+\omega} G_{i}(t, s) x_{i}(s) \\
& \quad \times\left[\sum_{j=1}^{n} a_{i j}(s) x_{j}(s)+\sum_{j=1}^{n} b_{i j}(s) \int_{-\tau_{i j}}^{0} f_{i j}(\xi) x_{j}(s+\xi) d \xi\right. \\
& \quad+\sum_{t \leq t_{k}<t+\omega} G_{i}\left(t, t_{k}\right) I_{i k}\left(x_{i}\left(t_{k}\right)\right) \\
& \leq \frac{1}{1-\delta_{i}}\left|x_{i}\right|_{0}\left\{\sum_{j=1}^{n} \int_{0}^{\omega}\left[a_{i j}(s)+b_{i j}(s)+c_{i j}(s)\right]\left|x_{j}\right|_{1} d s+\gamma_{i}\right\} \\
& \leq \frac{\beta_{i j} r+\gamma_{i}}{1-\delta_{i}}\left|x_{i}\right|_{0} \leq \delta_{i}\left|x_{i}\right|_{0}, \quad i=1,2, \ldots, n .
\end{aligned}
$$

In view of (42) and (43), we obtain

$$
\|x\|_{0} \leq\|T x\|_{0} \leq \max _{1 \leq i \leq n}\left\{\delta_{i}\right\}\|x\|_{0}<\|x\|_{0}
$$

which is a contradiction. Finally, we prove that $T x \not$ $x$, for all $x \in E$, and $\|x\|_{1}=R$ also hold. For this case, suppose, for the sake of contradiction, that there exist $x \in$ $E$ and $\|x\|_{1}=R$ such that $T x \leq x$. Furthermore, for any $t \in[0, \omega]$, we have

$$
\begin{aligned}
& x_{i}(t)-T_{i} x(t) \geq \delta_{i}\left|x_{i}-T_{i} x\right|_{1} \geq 0, \\
& \text { for any } t \in[0, \omega], i=1,2, \ldots, n .
\end{aligned}
$$

In addition, for any $t \in[0, \omega]$, we find

$$
\begin{aligned}
& \left(T_{i} x\right)(t) \\
& =\int_{t}^{t+\omega} G_{i}(t, s) x_{i}(s) \\
& \quad \times\left[\sum_{j=1}^{n} a_{i j}(s) x_{j}(s)+\sum_{j=1}^{n} b_{i j}(s) \int_{-\tau_{i j}}^{0} f_{i j}(\xi) x_{j}(s+\xi) d \xi\right. \\
& \left.\quad+\sum_{j \leq t_{k}<t+\omega} G_{i}\left(t, t_{k}\right) I_{i k}\left(x_{i}\left(t_{k}\right)\right) \int_{-\sigma_{i j}}^{0} g_{i j}(\xi) x_{j}^{\prime}(s+\xi) d \xi\right] d s \\
& >\frac{\delta_{i}^{2}}{1-\delta_{i}}\left|x_{i}\right|_{1} \sum_{j=1}^{n} \int_{0}^{\omega}\left[\delta_{j} a_{i j}(s)-b_{i j}(s)-c_{i j}(s)\right] d s\left|x_{j}\right|_{1} \\
& =\frac{\delta_{i}^{2}}{1-\delta_{i}}\left|x_{i}\right|_{1} \sum_{j=1}^{n}\left(\alpha_{i j}\left|x_{j}\right|_{1}\right), \quad i=1,2, \ldots, n .
\end{aligned}
$$


Thus, we have

$$
\begin{aligned}
\|T x\|_{0} & =\sum_{i=1}^{n}\left|T_{i} x\right|_{0}>\sum_{i=1}^{n} \frac{\delta_{i}^{2}}{1-\delta_{i}}\left|x_{i}\right|_{1} \sum_{j=1}^{n}\left(\alpha_{i j}\left|x_{j}\right|_{1}\right) \\
& \geq \min _{1 \leq i \leq n}\left\{\frac{\delta_{i}^{2} \min _{1 \leq j \leq n}\left\{\alpha_{i j}\right\}}{1-\delta_{i}}\right\} \sum_{i=1}^{n}\left|x_{i}\right|_{1} \sum_{j=1}^{n}\left|x_{j}\right|_{1} \\
& \geq \min _{1 \leq i \leq n}\left\{\frac{\delta_{i}^{2} \min _{1 \leq j \leq n}\left\{\alpha_{i j}\right\}}{1-\delta_{i}}\right\} R^{2}=R .
\end{aligned}
$$

From (45) and (47), we obtain

$$
\|x\|_{1} \geq\|T x\|_{1} \geq\|T x\|_{0}>R
$$

which is a contradiction. Therefore, condition (ii) of Lemma 7 holds. By Lemma 7, we see that $T$ has at least one nonzero fixed point in $E$. Thus, the system (6) has at least one positive $\omega$-periodic solution. Therefore, it follows from Lemma 6 that system (2) has a positive $\omega$-periodic solution. The proof of Theorem 11 is complete.

Remark 12. If $I_{i k}\left(x\left(t_{k}\right)\right)=0$, we can easily derive the corresponding results in [10]. So we extend the corresponding results in [10].

\section{Example}

In this section, we give an example to show the effectiveness of our result.

Example 1. Consider the following nonimpulsive system:

$$
\begin{aligned}
& x_{1}^{\prime}(t) \\
& =x_{1}(t)\left[\frac{1-\sin t}{24}-(10+\sin t) x_{1}(t)\right. \\
& -(9-\cos t) x_{2}(t)-(3-2 \cos t) \\
& \quad \times \int_{-\tau_{11}}^{0} f_{11}(\xi) x_{1}(t+\xi) d \xi-\frac{5+\sin t}{15} \\
& \quad \times \int_{-\tau_{12}}^{0} f_{12}(\xi) x_{2}(t+\xi) d \xi-(1+\sin t) \\
& \quad \times \int_{-\sigma_{11}}^{0} g_{11}(\xi) x_{1}^{\prime}(t+\xi) d \xi-\frac{4-3 \cos t}{6} \\
& \left.\quad \times \int_{-\sigma_{12}}^{0} g_{12}(\xi) x_{2}^{\prime}(t+\xi) d \xi\right],
\end{aligned}
$$

$$
\begin{aligned}
& x_{2}^{\prime}(t) \\
&=x_{2}(t)\left[\frac{1+\cos t}{8 \pi}-(12+3 \cos t) x_{1}(t)\right. \\
&-(7-\sin t) x_{2}(t)-(2+3 \sin t) \\
& \times \int_{-\tau_{21}}^{0} f_{21}(\xi) x_{2}(t+\xi) d \xi-(1-\cos t) \\
& \times \int_{-\tau_{22}}^{0} f_{22}(\xi) x_{2}(t+\xi) d \xi-\frac{4+\sin t}{12} \\
& \times \int_{-\sigma_{21}}^{0} g_{21}(\xi) x_{2}^{\prime}(t+\xi) d \xi-\frac{5-\cos t}{10} \\
&\left.\times \int_{-\sigma_{22}}^{0} g_{22}(\xi) x_{2}^{\prime}(t+\xi) d \xi\right],
\end{aligned}
$$

where $\tau_{i j}, \sigma_{i j} \in(0,+\infty)(i, j=1,2)$, and $f_{i j}, g_{i j} \in$ $C\left(R, R^{+}\right)$satisfying $\int_{-\tau_{i j}}^{0} f_{i j}(\xi) d \xi=1, \int_{-\sigma_{i j}}^{0} g_{i j}(\xi) d \xi, i, j=1,2$. Obviously

$$
\begin{array}{r}
r_{1}(t)=\frac{1-\sin t}{24}, \quad r_{2}=\frac{1+\cos t}{8 \pi}, \\
a_{11}(t)=10+\sin t, \\
a_{12}(t)=9-\cos t, \quad a_{21}(t)=12+3 \cos t, \\
a_{22}(t)=7-\sin t, \quad b_{11}(t)=3-2 \cos t,
\end{array}
$$

$$
b_{12}(t)=\frac{5+\sin t}{15},
$$

$$
\begin{array}{r}
b_{21}(t)=2+3 \sin t, \quad b_{22}(t)=1-\cos t, \\
c_{11}(t)=1+\sin t, \quad c_{12}(t)=\frac{4-3 \cos t}{6}, \\
c_{21}(t)=\frac{4+\sin t}{12}, \\
c_{22}(t)=\frac{5-\cos t}{10}, \quad r_{1}^{L}=r_{2}^{L}=0 .
\end{array}
$$

Furthermore, we obtain

$$
\begin{gathered}
\delta_{1}=e^{-(\pi / 12)}<1, \quad \delta_{2}=e^{-(1 / 4)}<1, \\
\alpha_{11}=20 \pi \delta_{1}-8 \pi \approx 23.8007, \\
\alpha_{12}=18 \pi \delta_{2}-2 \pi \approx 37.2423, \\
\alpha_{21}=24 \pi \delta_{1}-\frac{14 \pi}{3} \approx 44.0594, \\
\alpha_{22}=14 \pi \delta_{2}-3 \pi \approx 24.4284,
\end{gathered}
$$




$$
\begin{aligned}
& \min _{1 \leq j \leq 2}\left\{\alpha_{1 j}\right\}=\alpha_{11}=20 \pi \delta_{1}-8 \pi \approx 23.8007, \\
& \min _{1 \leq j \leq 2}\left\{\alpha_{2 j}\right\}=\alpha_{22}=14 \pi \delta_{2}-3 \pi \approx 24.4284, \\
& \delta_{1} a_{11}(t)-b_{11}(t)-c_{11}(t)>0.009>0, \\
& \delta_{2} a_{12}(t)-b_{12}(t)-c_{12}(t)>1>0, \\
& \delta_{1} a_{21}(t)-b_{21}(t)-c_{21}(t)>4>0, \\
& \delta_{2} a_{22}(t)-b_{22}(t)-c_{22}(t)>2>0 \text {, } \\
& \left(1+r_{1}^{L}\right) \frac{\delta_{1}^{2} \alpha_{11}}{1-\delta_{1}}=\frac{e^{-(\pi / 6)}\left(20 \pi \delta_{1}-8 \pi\right)}{1-e^{-(\pi / 12)}} \\
& \approx 65.2614>18 \\
& \geq \max _{0 \leq t \leq 2 \pi}\left\{a_{11}(t)+b_{11}(t)+c_{11}(t)\right\}, \\
& \left(1+r_{1}^{L}\right) \frac{\delta_{1}^{2} \alpha_{12}}{1-\delta_{1}}=\frac{e^{-(\pi / 6)}\left(18 \pi \delta_{2}-2 \pi\right)}{1-e^{-(\pi / 12)}} \\
& \approx 102.1184>\frac{347}{30} \\
& \geq \max _{0 \leq t \leq 2 \pi}\left\{a_{12}(t)+b_{12}(t)+c_{12}(t)\right\}, \\
& \left(1+r_{2}^{L}\right) \frac{\delta_{2}^{2} \alpha_{21}}{1-\delta_{2}}=\frac{e^{-(1 / 2)}\left(24 \pi \delta_{1}-(14 \pi / 3)\right)}{1-e^{-(1 / 4)}} \\
& \approx 113.3411>\frac{245}{12} \\
& >\max _{0 \leq t \leq 2 \pi}\left\{a_{21}(t)+b_{21}(t)+c_{21}(t)\right\}, \\
& \left(1+r_{2}^{L}\right) \frac{\delta_{2}^{2} \alpha_{22}}{1-\delta_{2}}=\frac{e^{-(1 / 2)}\left(14 \pi \delta_{2}-3 \pi\right)}{1-e^{-(1 / 4)}} \\
& \approx 62.8411>\frac{53}{5} \\
& >\max _{0 \leq t \leq 2 \pi}\left\{a_{22}(t)+b_{22}(t)+c_{22}(t)\right\}, \\
& \frac{\delta_{1}^{2} \min _{1 \leq j \leq 2}\left\{\alpha_{1 j}\right\}}{1-\delta_{1}}=\frac{e^{-(\pi / 6)}\left(20 \pi \delta_{1}-6 \pi\right)}{1-e^{-(\pi / 12)}} \approx 65.2614, \\
& \frac{\delta_{2}^{2} \min _{1 \leq j \leq 2}\left\{\alpha_{2 j}\right\}}{1-\delta_{2}}=\frac{e^{-(1 / 2)}\left(14 \pi \delta_{2}-3 \pi\right)}{1-e^{-(1 / 4)}} \approx 62.8411 \text {, } \\
& \min _{1 \leq i \leq 2}\left\{\frac{\delta_{i}^{2} \min _{1 \leq j \leq 2}\left\{\alpha_{i j}\right\}}{1-\delta_{i}}\right\}=\frac{\delta_{2}^{2} \min _{1 \leq j \leq 2}\left\{\alpha_{2 j}\right\}}{1-\delta_{1}} \approx 62.8411 \text {, } \\
& c_{11}^{M}+c_{12}^{M}=\frac{19}{6}, \quad c_{21}^{M}+c_{22}^{M}=\frac{61}{60}, \\
& \max _{1 \leq i \leq 2}\left\{\sum_{j=1}^{2} d_{i j}^{M}\right\}=\frac{19}{6} \text {. }
\end{aligned}
$$

Therefore,

$$
\frac{19}{6}=\max _{1 \leq i \leq 2}\left\{\sum_{i=1}^{2} d_{i j}^{M}\right\}<\min _{1 \leq i \leq 2}\left\{\frac{\delta_{i}^{2} \min _{1 \leq j \leq 2}\left\{\alpha_{i j}\right\}}{1-\delta_{i}}\right\} \approx 62.8411 .
$$

Hence, $\left(A_{1}\right)-\left(A_{3}\right),\left(A_{5}\right)$ hold and $a_{i}^{M} \leq 1, i=1,2$. According to Theorem 11, system (49) has at least one positive $2 \pi$-periodic solution.

\section{Acknowledgments}

The authors are thankful to the referees and editor for the improvement of the paper. This work was supported by the Construct Program of the Key Discipline in Hunan Province. Research was supported by the National Natural Science Foundation of China $(10971229,11161015)$, the China Postdoctoral Science Foundation (2012M512162), and Hunan Provincial Natural Science-Hengyang United Foundation of China (11JJ9002).

\section{References}

[1] P. Chesson, "Understanding the role of environmental variation in population and community dynamics," Theoretical Population Biology, vol. 64, no. 3, pp. 253-254, 2003.

[2] J. Zhen and Z. E. Ma, "Periodic solutions for delay differential equations model of plankton allelopathy," Computers \& Mathematics with Applications, vol. 44, no. 3-4, pp. 491-500, 2002.

[3] X. Liu and G. Ballinger, "Boundedness for impulsive delay differential equations and applications to population growth models," Nonlinear Analysis: Theory, Methods \& Applications, vol. 53, no. 7-8, pp. 1041-1062, 2003.

[4] Y. Kuang, Delay Differential Equations with Applications in Population Dynamics, Academic Press, New York, NY, USA, 1993.

[5] Y. K. Li and Y. Kuang, "Periodic solutions of periodic delay Lotka-Volterra equations and systems," Journal of Mathematical Analysis and Applications, vol. 255, no. 1, pp. 260-280, 2001.

[6] Y. K. Li and L. F. Zhu, "Existence of periodic solutions of discrete Lotka-Volterra systems with delays," Bulletin of the Institute of Mathematics, Academia Sinica, vol. 33, no. 4, pp. 369-380, 2005.

[7] H. Zhao and N. Ding, "Existence and global attractivity of positive periodic solution for competition-predator system with variable delays," Chaos, Solitons \& Fractals, vol. 29, no. 1, pp. 162170, 2006.

[8] Y. Song, M. Han, and Y. Peng, "Stability and Hopf bifurcations in a competitive Lotka-Volterra system with two delays," Chaos, Solitons \& Fractals, vol. 22, no. 5, pp. 1139-1148, 2004.

[9] Z. Yang and J. Cao, "Positive periodic solutions of neutral LotkaVolterra system with periodic delays," Applied Mathematics and Computation, vol. 149, no. 3, pp. 661-687, 2004.

[10] Y. K. Li, "Positive periodic solutions of periodic neutral LotkaVolterra system with distributed delays," Chaos, Solitons \& Fractals, vol. 37, no. 1, pp. 288-298, 2008.

[11] A. M. Samoikleno and N. A. Perestyuk, Impulsive Differential Equations, World Scientific, Singapore, 1995.

[12] S. T. Zavalishchin and A. N. Sesekin, Dynamic Impulse Systems, Theory and Applications, Kluwer Academic Publishers, Dordrecht, The Netherlands, 1997. 
[13] V. Lakshmikantham, D. D. Bainov, and P. S. Simenov, Theory of Impulsive Differential Equations, World Science, Singapore, 1989.

[14] J. R. Yan, A. P. Zhao, and J. J. Nieto, "Existence and global attractivity of positive periodic solution of periodic singlespecies impulsive Lotka-Volterra systems," Mathematical and Computer Modelling, vol. 40, no. 5-6, pp. 509-518, 2004.

[15] X. Liu and G. Ballinger, "Boundedness for impulsive delay differential equations and applications to population growth models," Nonlinear Analysis: Theory, Methods \& Applications, vol. 53, no. 7-8, pp. 1041-1062, 2003.

[16] W. Zhang and M. Fan, "Periodicity in a generalized ecological competition system governed by impulsive differential equations with delays," Mathematical and Computer Modelling, vol. 39, no. 4-5, pp. 479-493, 2004.

[17] S. L. Sun and L. S. Chen, "Existence of positive periodic solution of an impulsive delay logistic model," Applied Mathematics and Computation, vol. 184, no. 2, pp. 617-623, 2007.

[18] X. Li, X. Lin, D. Jiang, and X. Zhang, "Existence and multiplicity of positive periodic solutions to functional differential equations with impulse effects," Nonlinear Analysis: Theory, Methods \& Applications, vol. 62, no. 4, pp. 683-701, 2005.

[19] R. E. Gaines and J. L. Mawhin, Coincidence Degree Theory and Nonlinear Differential Equations, Springer, Berlin, Germany, 1977.

[20] N. P. Các and J. A. Gatica, "Fixed point theorems for mappings in ordered Banach spaces," Journal of Mathematical Analysis and Applications, vol. 71, no. 2, pp. 547-557, 1979.

[21] D. J. Guo, Nonlinear Functional Analysis, in Chinese, ShanDong Science and Technology Press, 2001.

[22] D. Bainov and P. Simeonov, Impulsive Differential Equations: Periodic Solutions and Applications, vol. 66 of Pitman Monographs and Surveys in Pure and Applied Mathematics, 1993.

[23] D. J. Guo, "Positive solutions to nonlinear operator equations and their applications to nonlinear integral equations," Advances in Mathematics, vol. 13, no. 4, pp. 294-310, 1984 (Chinese). 


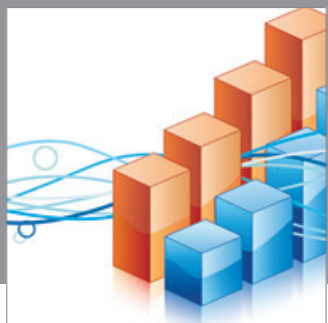

Advances in

Operations Research

mansans

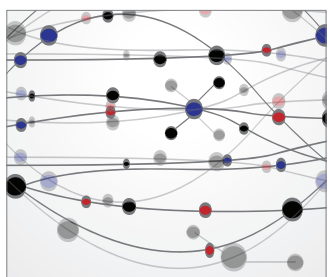

The Scientific World Journal
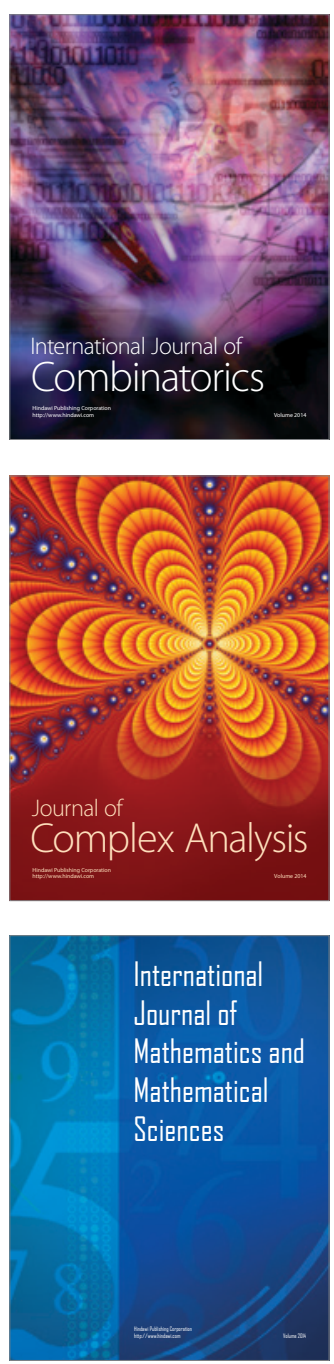
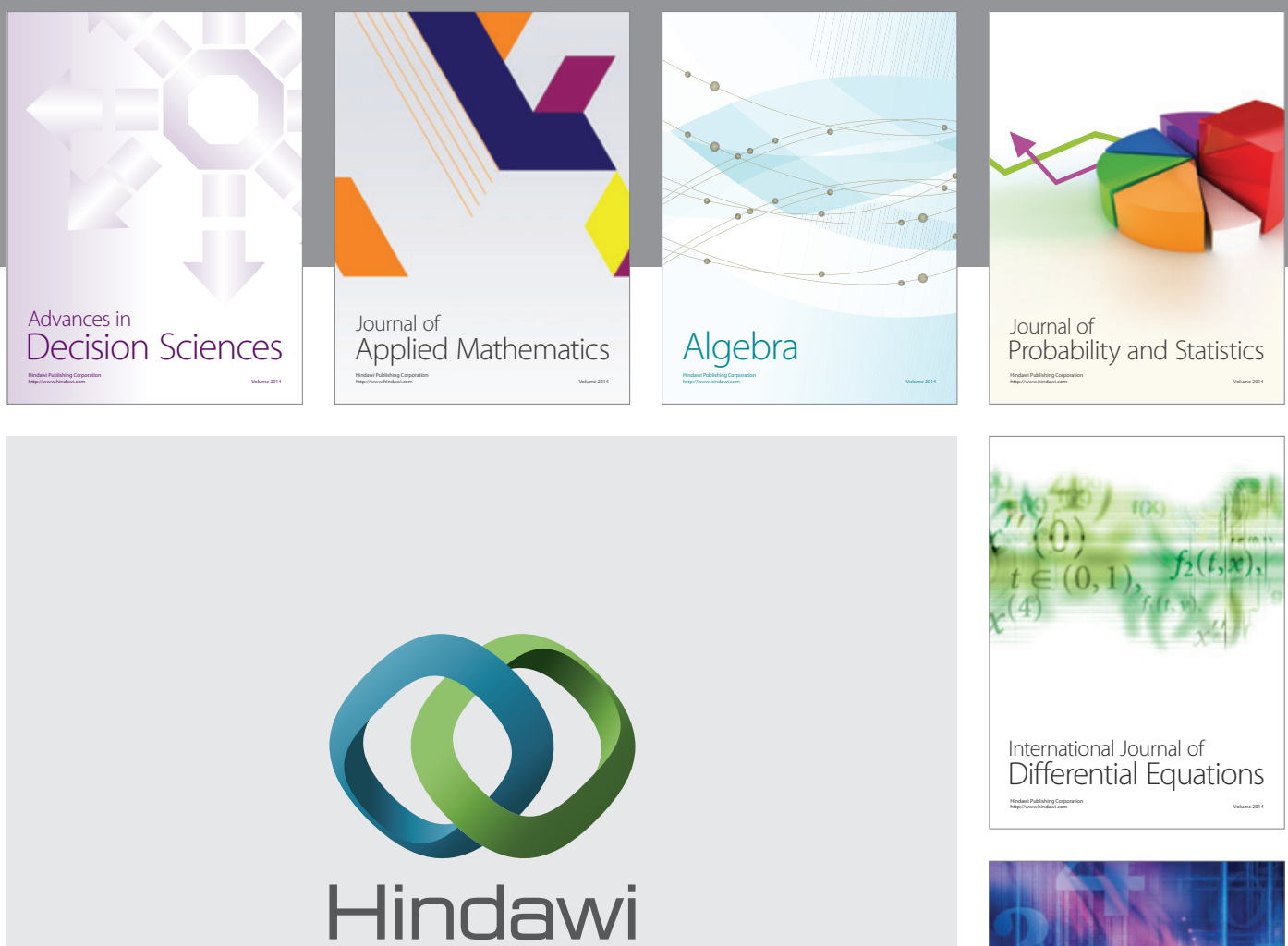

Submit your manuscripts at http://www.hindawi.com
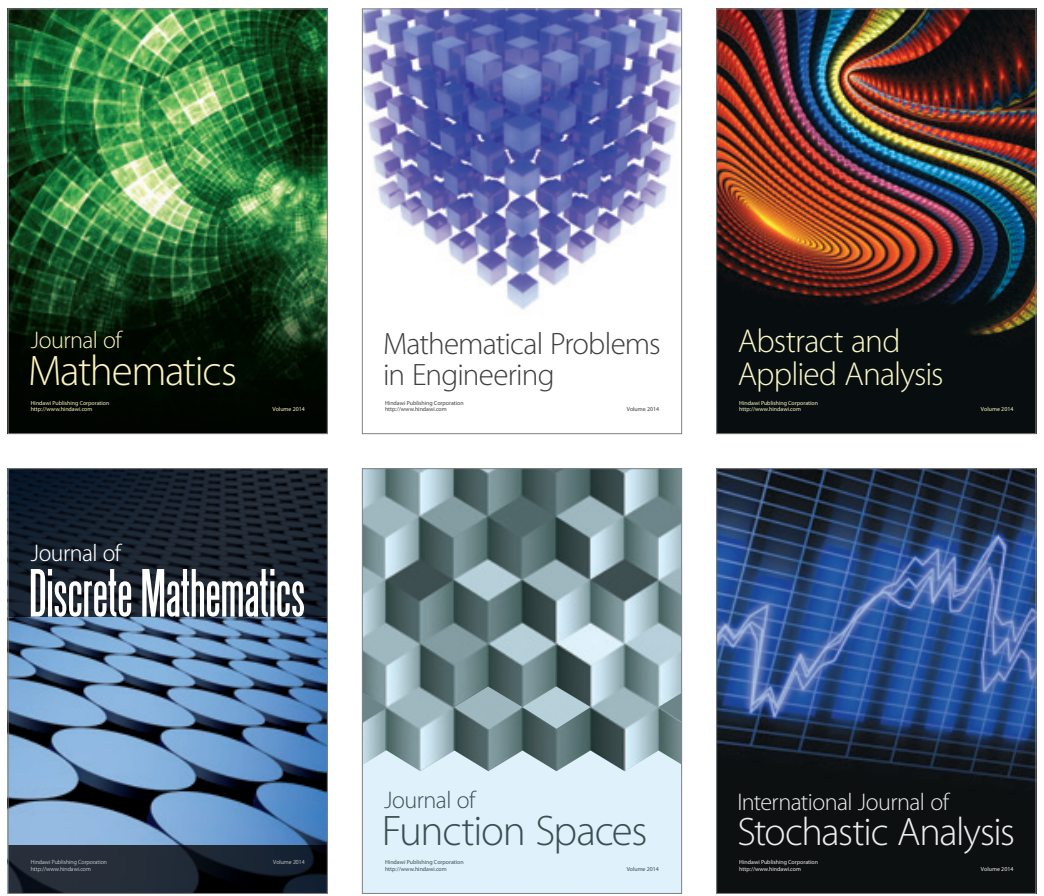

Journal of

Function Spaces

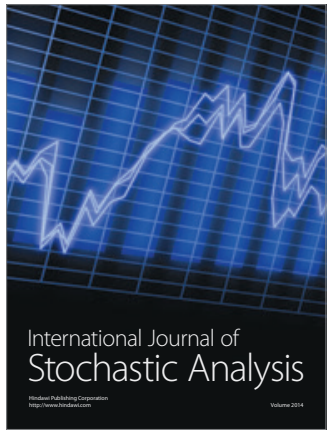

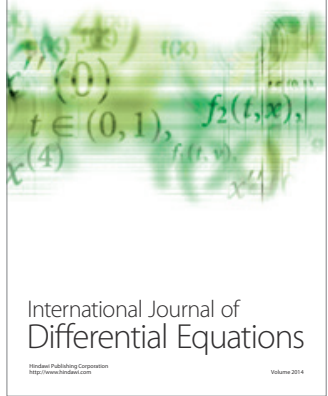
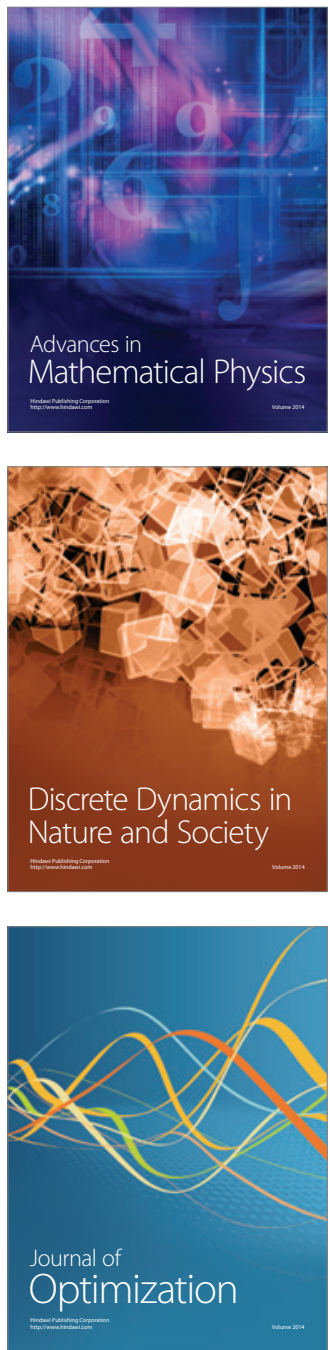